\title{
Comparison of Hydrogen Bonding for Chiral and Racemic 1-Monostearoylglycerols in Solvent
}

\author{
Hideyo Matsuzawa*, Kiyofumi Murakami and Makio Iwahashi \\ Department of Chemistry, School of Science, Kitasato University (1-15-1 Kitasato, Sagamihara, Kanagawa-ken 228-8555, JAPAN)
}

\begin{abstract}
Comparison of the hydrogen bonding of racemic RS-1-monostearoylglycerol and chiral $S$-1monostearoylglycerol in benzene was carried out through the NMR measurement. In addition, the concentration dependence of a chirality of $S$-1-monostearoylglycerol in hexane was studied though UV and circular dichroism (CD) measurements. The chemical shifts of OH protons of the $S$ - and $R S$-1monostearoylglycerols indicated that the hydrogen bonding between the $R$ - and $S$-form molecules of $R S$-1monostearoylglycerol is stronger than that between the $S$ - and $S$-form molecules of $S$-1-monostearoylglycerol in the low concentration region and that the difference in the strength of hydrogen-bonding between them becomes small in the high concentration region. The chirality of the $S$-1-monostearoyglycerol in hexane decreased with increasing its concentration. This suggests that the association of chiral $S$-1-monoacylglycerol arising from its increasing concentration reduces the chirality of $S$-1-monostearoylglycerol.
\end{abstract}

Key words: hydrogen bonding, chiral 1-monostearoyl glycerol, racemic 1-monostearoyl glycerol, NMR chemical shift, Cicular dichroism

\section{INTRODUCTION}

Triacylglycerols composed of three acylgroups and one glycerol are the main component of natural oils and fats. In nature there also exist monoacylglycerols in which only one acylgroup combines with one glycerol.

The monoacylglycerols, having one hydrophobic moiety and two hydrophilic $\mathrm{OH}$ moieties, show strong surfaceactive ability. Hence, they are used as superior surfactants being safe for living organisms, e.g., for toiletries, medicine and pharmaceuticals and food supplies.

Two types of monoacylglycerols exist depending on the acylgroup position combining to glycerol, i.e., 1-monoacylglycerol and 2-monoacylgrycerol. The former has an asymmetric carbon atom, whereas the latter does not. Thus the former should have two chiral structures, i.e., $R$ and $S$-forms. In general, however, only $S$-form and racemic $R S$-form are commercially available as the $1^{-}$ monoacylglycerol having hydrocarbon chains with the length from $\mathrm{C}_{12}$ to $\mathrm{C}_{18}$.

Properties of the 1-monoacylglycerol especially for its $R S$-form have been studied by many investigators ${ }^{1-6)}$. How ${ }^{-}$ ever, little has yet been reported on the physicochemical difference between optically active $S$-form and inactive
$R S$-form.

The racemic $R S$-form in the crystalline state can be classified, in terms of the interaction of $R$ - and $S$-molecules in the crystal, into three types of modification: a racemic compound, a solid solution and a simple mixture of $R$ - and $S$-form molecules ${ }^{7,8}$. $R S$-1-monoacylgryserols containing carbon-chains from $\mathrm{C}_{12}$ to $\mathrm{C}_{18}$ are thought to be racemic compounds. This is because the $R S$-1-monoacylgryserols have higher melting points than their optically active $S$ forms ${ }^{9}$. Thus the various crystalline properties of the racemic compounds are expected to differ from those of the optically active species. The thermodynamic and structural properties of the crystals of $R S$-1-monoacylglycerol were compared with those of $S$-1-monoacylglycerol, ${ }^{9,10)}$. Through the measurements of DSC, solubility in benzene, IR spectra and X-ray diffraction for $S$ - and $R S$-forms of 1monostearoylglycerol and 1-monopalmitoylglycerol it was assumed that their $R S$-forms are the racemic compounds in their melts, in which the $R$ - and $S$-form molecules are strongly combined with each other, and that in a solvent the interaction between the molecules of $R$ - and $S$-forms is not so strong that the molecules are free from each other ${ }^{10}$.

Recently, however, the near infrared spectroscopic mea-

*Correspondence to: Hideyo Matsuzawa, Department of Chemistry, School of Science, Kitasato University, 1-15-1 Kitasato, Sagamihara, Kanagawa-ken 228-8555, JAPAN

E-mail: matsu@sci.kitasato-u.ac.jp

Accepted January 29, 2008 (received for review December 3, 2007)

Journal of Oleo Science ISSN 1345-8957 print / ISSN 1347-3352 online

http://www.jstage.jst.go.jp/browse/jos/ 


\section{H. Matsuzawa, K. Murakami and M. Iwahashi}

surements for alcohols in solvents indicated that the alcohol molecules associate to form their dimers, trimers and oligomers even at relatively low concentration such as 0.1 mol dm${ }^{-311,12)}$. Furthermore the fatty acid in $\mathrm{CCl}_{4}$ associates to form dimer even at extremely low concentration such as $10^{-2} \sim 10^{-3} \mathrm{~mol} \mathrm{dm}^{-313)}$. Thus the 1-monoacylglycerol having two $\mathrm{OH}$ groups would more easily combine with each other by their strong hydrogen bonding in the solvents.

In the present study, the difference in the hydrogen bonding between the $S$ - and $R S$-1-monostearoylglycerols in benzene was studied through the measurement of ${ }^{1} \mathrm{H}$ NMR. The concentration dependence of chirality of $S$-1-monostearoyglycerols in hexane was also studied through the measurements of UV and circular dichroism (CD).

\section{EXPERIMENTAL}

\subsection{Materials}

Samples ( $\gg 99 \%$ pure) of optically active $S$ - and racemic $R S$-1-monostearoylglycerols obtained from Fluka AG (Switzerland) were purified by repeated recrystallization from fleshly distillated hexane. Hexane (Junsei Kagaku Co., ultra-analytical grade) used for the recrystallization and the UV and CD measurements were prepared by distillation after overnight drying with $\mathrm{CaCl}_{2}$ and four hours refluxing with metallic sodium. Benzene (Junsei Kagaku Co., spectroscopic grade) for DSC measurements was prepared by distillation after overnight drying with $\mathrm{CaCl}_{2}$ and four hours refluxing with $\mathrm{CaH}_{2}$. Deuterated benzene (atomic purity $99.8 \%$, Merck (Tokyo)) was used without further purification.

The heat of fusion and the melting temperature were determined on a differential scanning calorimeter (Rigaku Denki, model 8230B) in the temperature range of 300-373 K at a constant rate of $5 \mathrm{~K} \mathrm{~min}^{-1}$ to rise the temperature. In order to obtain melting temperature-composition diagrams for the mixtures of $S$ - and $R S$-1-monostearoyglycerols, they were mixed in benzene and allowed to stand overnight under a reduced pressure to evaporate the benzene.

The UV-absorption and CD spectra were recorded on a Hitachi U-3210 spectrophotometer and a JASCO J-500 spectropolarimeter, respectively, both of which were equipped with a temperature-control unit.

The ${ }^{1} \mathrm{H}$ NMR spectra were recorded on a JEOL Model EX 400 NMR spectrometer with a sample tube of $5.0 \mathrm{~mm}$ diameter at $25,35,50,60$ and $75 \pm 0.5^{\circ} \mathrm{C}$. The hydroxyl proton chemical shifts, relative to the proton of benzene existing in the deuterated benzene that is used for locking purposes, were measured as a function of the concentration of 1-monostearolglycerol. The deuterated benzene was contained in a coaxial tube.

\section{RESULTS AND DISCUSSION}

\subsection{Phase diagram}

DSC thermograms were obtained by heating scan for pure $S$-form, pure $R S$-form and $S$-/RS-form mixtures with several compositions. The temperatures of the endothermic peaks of DSC thermograms define the phase boundaries. Thus, the phase diagram of the binary mixture can be constructed by plotting the DSC peak temperatures against the composition. The phase diagram thus obtained for $S$-/RS-form mixture of 1-monostearoylglycerol is shown in Fig. 1, where the composition is expressed in terms of the mole fraction of $R S$-form, $x_{\mathrm{RS}}$. This phase diagram exhibits a typical eutectic pattern: $R S-1$-monostearoylglycerol is the racemic compound. Thus, the horizontal solidus line extending over the whole composition range suggests that $S$ - and $R S$-forms are completely immiscible and exist as separate phases in a solid phase.

Melting temperatures of $S$-form and $R S$-form were 349.3 and $355.6 \mathrm{~K}$, respectively, which are in good agreement with the corresponding values available from literature, $349.2 \mathrm{~K}$ for $S$ - and $355.2 \mathrm{~K}$ for $R S$-forms ( $\beta$-form crystals $)^{9,10)}$. The obtained heat of fusion for $S$-form and $R S$-form were $82.4 \mathrm{kJmol}^{-1}$ and $75.4 \mathrm{kJmol}^{-1}$, respectively, which are in agreement with the values in literature ${ }^{10}$. Thus, we reconfirmed that $R S$-form has a higher melting temperature and lower heat of fusion than the $S$-form,. This anomaly in the order of their values between melting temperatures and heat of fusion is explainable by assuming that the interaction between $R$ - and $S$-form molecules is stronger than that between $S$ - and $S$-form molecules and that $R S$-form

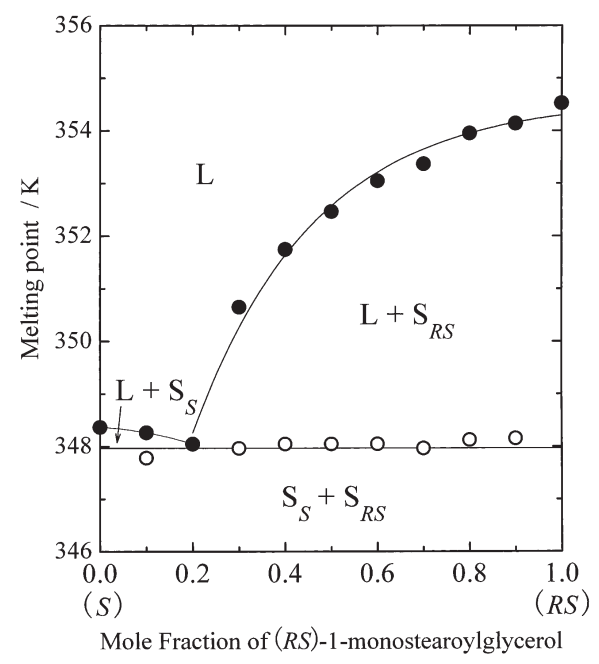

Fig. 1 Phase Diagram of the Binary Mixture of $S$ - and $R S$ 1- Monostearoylglycerols.

The phase diagram was constructed by plotting the peak temperatures of DSC thermogram against the mole fraction of $R S$-1-monostearoylglycerol, $x_{\mathrm{RS}}$, in the mixture. 
crystal does not sufficiently dissociate into completely free $R$ - and $S$-form molecules in a liquid just above the melting temperature. Namely, there would remain the more amount of $R-S$ compound than the $S-S$ compound in their liquid states.

3.2 Comparison of hydrogen bondings of RS- and S-1monostearoylglycerols in benzene by their ${ }^{1} \mathrm{H}$ NMR chemical shifts

In order to compare the strength of the interaction between $R$ - and $S$-form molecules with that between $S$ and $S$-form molecules in solvent, we carried out the ${ }^{1} \mathrm{H}$ NMR measurement for $R S$ - and $S$-1-monostearoylglycerols in benzene; in this ${ }^{1} \mathrm{H}$ NMR experiment we used benzene$\mathrm{d}_{6}$. This is because the solbility of $R S$ - and $S$-1-monostearoylglycerols in benzene is relatively high at room temperature, whereas hexane is a poor solvent for them. The chemical shift assignments were carried out using the proton signal $(7.20 \mathrm{ppm})$ of benzene as a reference, which exists slightly in benzene- $\mathrm{d}_{6}$. Figure 2 shows a typical NMR spectrum for $R S$-1-monostearoylglycerol $\left(4.46 \times 10^{-3}\right.$ mol dm$\left.{ }^{-3}\right)$ in benzene- $\mathrm{d}_{6}$ at $25^{\circ} \mathrm{C}$. There exists a proton signal of $\mathrm{OH}$ moiety at $1.950 \mathrm{ppm}$. The chemical shift of each signal except the proton signal of $\mathrm{OH}$ moiety was held constant regardless of the temperature and the concentration of $R S$ - or $S$-1-monostearoylglycerol. $R S$ - and $S$-1-monostearoylglycerol gave almost same signal pattern except for the $\mathrm{OH}$ proton signal.

Figure 3 shows the temperature and concentration dependence of the chemical shifts of $\mathrm{OH}$ proton for $R S-1-$ monostearoylglycerol $(\bigcirc)$ and $S$-1-monostearoylglycerol $(\boldsymbol{\Delta})$ in benzene- $\mathrm{d}_{6}$. At each constant temperature the both chemical shifts, $\boldsymbol{\delta}_{\mathrm{OH}}$, of protons in $\mathrm{OH}$ moieties of $R S$-1- and
$S$-1-monostearoylglycerols increase with increasing their concentrations; the $\delta_{\mathrm{OH}}$ values of $R S$-1-monostearoylglycerol are, as a whole, larger than those of $S$-1-monostearoylglycerol. On the other hand, at each constant concentration of the $S$ - or $R S$-1-monostearoylglycerol, the $\delta_{\mathrm{OH}}$ of $S$ or $R S$-1-monostearoylglycerol decreases with increasing temperature. The proton-signal shift to higher magnetic field with temperature would be attributable to the dissociation of the hydrogen-bonding aggregate, whereas the shift to lower magnetic field with concentration, to the forma-

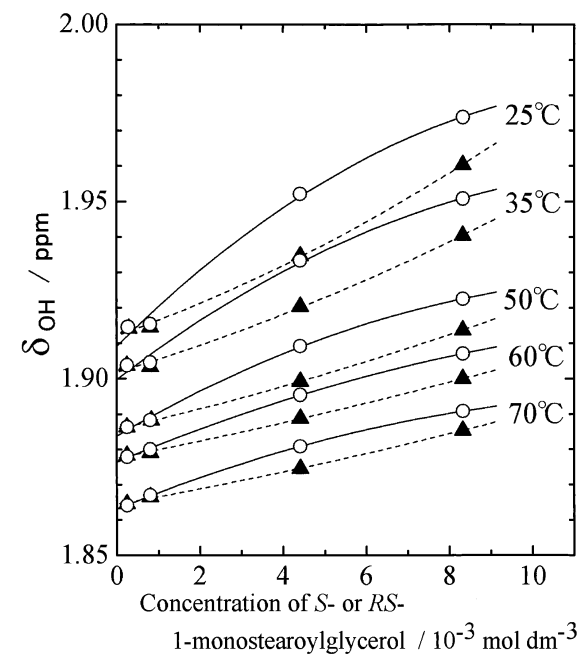

Fig. 3 Chemical Shift versus Concentration Relationship for O-H Proton, $\delta_{\mathrm{OH}}$, in $S$-form ( $(\boldsymbol{\Delta})$ and $R S$-Form ( $\bigcirc$ ) of 1-Monostearoylglycerol at Various Temperatures $\left(25,35,50,60\right.$ and $\left.75^{\circ} \mathrm{C}\right)$.

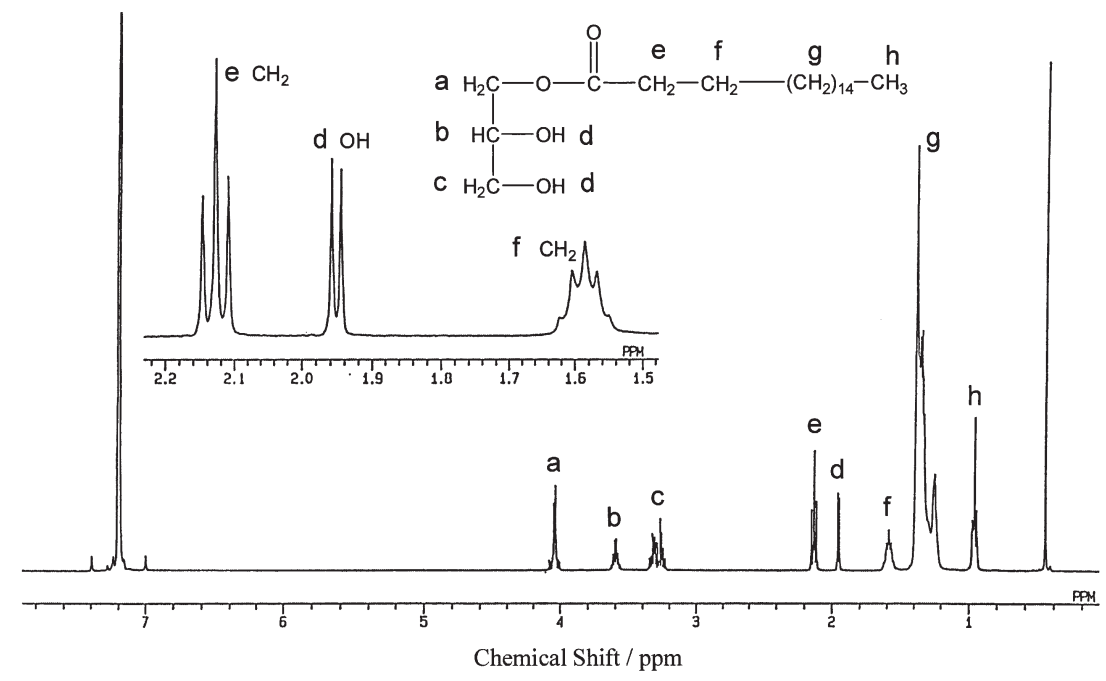

Fig. $2{ }^{1} \mathrm{H}$ NMR Spectrum of $4.46 \times 10^{-3} \mathrm{~mol} \mathrm{dm}^{-3} R S$-1-Monostearoylglycerol in Benzene- $\mathrm{d}_{6}$ at $25^{\circ} \mathrm{C}$. 
tion of the hydrogen-bonding aggregate. The $\delta_{\mathrm{OH}}$ of $S$-form and that of $R S$-form are in agreement with each other at low concentration $\left(2.79 \times 10^{-4} \mathrm{~mol} \mathrm{dm}^{-3}\right)$ and a high temperature $\left(70^{\circ} \mathrm{C}\right)$. Probably, under the condition such as low concentration and high temperature, $R$ - and $S$-form molecules would exist as free monomers even in the $R S-1$ monostearoylglyserol solution. Thus, from the results of NMR, it is concluded that the hydrogen-bonding interaction between $R$ - and $S$-molecules of 1-monosteroylglycerol is slightly stronger than that between $S$ - and $S$-molecules in benzene.

\subsection{Effect of concentration of S-1-monostearoylglycerol in hexane on its chirality}

In order to know the effect of the concentration of $S$-1monostearoylglycerol in a solvent on its chirality, we carried out the UV and $\mathrm{CD}$ measurements. In this experiment we used hexane at high temperature $\left(63.1^{\circ} \mathrm{C}\right)$. This is because benzene has a strong absorption band which interferes the observation of $\mathrm{C}=\mathrm{O}$ band of $S$-1-monostearoylglycerol in its UV spectrum, whereas hexane has no such interference.

Figure 4 shows the concentration dependence of UVabsorption and CD spectra for $S$-1-monostearoylglycerol in hexane at $63.1^{\circ} \mathrm{C}$. The UV-absorption and CD spectra have a band having a peak around $214 \mathrm{~nm}$. The intensity of the UV-absorption and CD band-peaks increases with increasing concentration. The bands in both UV-absorption and $\mathrm{CD}$ spectra are due to the $\left(\mathrm{n}, \pi^{*}\right)$ transition of $\mathrm{C}=\mathrm{O}$ bond ${ }^{14,15)}$. The $R S$-form gave almost the same UV spectra as $S$-form; the CD spectra for $R S$-1-monostearoylglycerol were, of

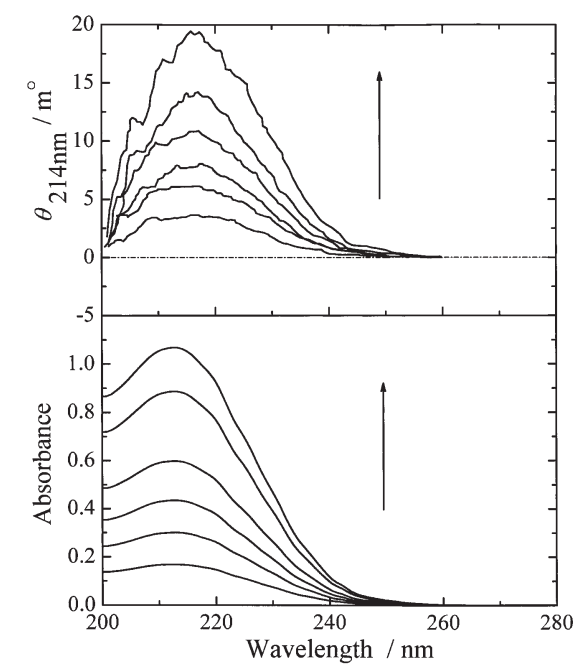

Fig. 4 Concentration Dependences of UV and CD Spectra of $S$-1-Monostearoylglycerol in Hexane at $63.1^{\circ} \mathrm{C}$. The concentrations for curves from bottom to top are $3.01 \times 10^{-3}, 5.30 \times 10^{-3}, 7.70 \times 10^{-3}, 1.01 \times 10^{-2}$, $1.46 \times 10^{-2}$ and $1.87 \times 10^{-2} \mathrm{~mol} \mathrm{dm}^{-3}$. course, not observed.

Figure 5 shows the absorbance versus concentration relationships at $214 \mathrm{~nm}$ for $R S$ - and $S$-forms. The two relationships are in fair agreement with each other and indicate a good linearity. Thus Lambert-Beer low is applicable to the $R S$ - and $S$-forms in this concentration range. From the slop of the line, we obtained the molar absorption coefficient, $\varepsilon\left(=60 \mathrm{~mol}^{-1} \mathrm{dm}^{3} \mathrm{~cm}^{-1}\right)$, for the absorption band at $214 \mathrm{~nm}$ for $S$-1-monostearoylglycerol.

Figure 6 shows the concentration dependence of the ellipticity at $214 \mathrm{~nm}, \theta_{214 \mathrm{~nm}} / \mathrm{m}^{\circ}$, of $S$-1-monostearoylglycerol

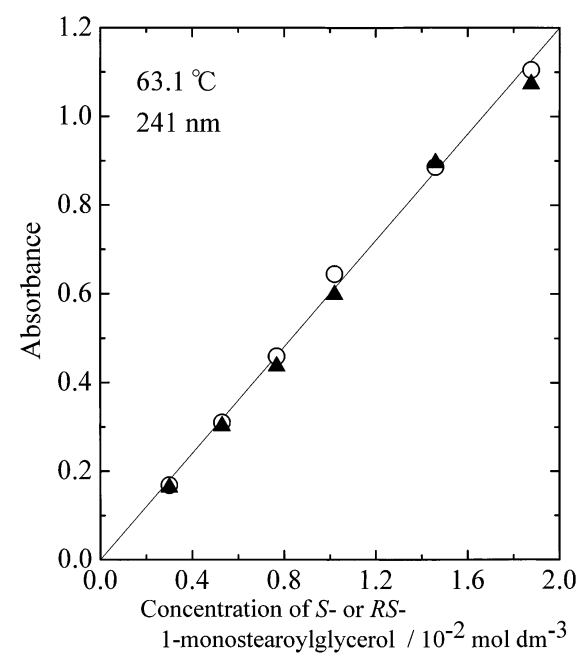

Fig. 5 Absorbance at $214 \mathrm{~nm}$ versus Cconcentration Relationship for $S$-Form $(\boldsymbol{A})$ and $R S$-Form $(\bigcirc)$ at $63.1^{\circ} \mathrm{C}$.

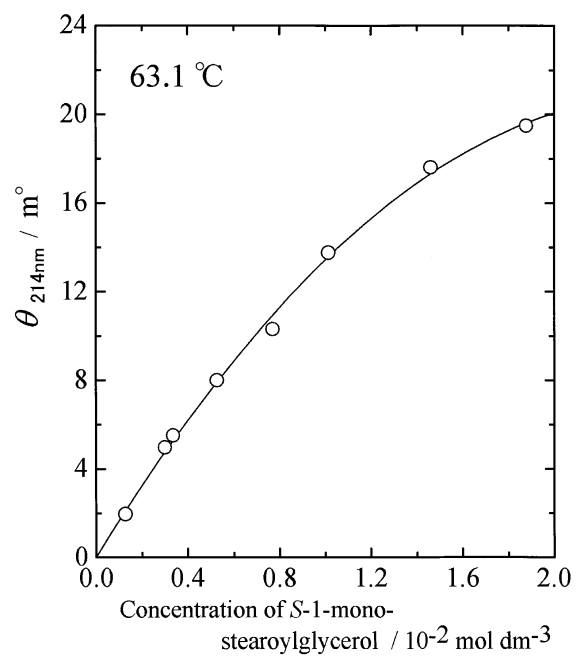

Fig. 6 Concentration Dependence of the Ellipticity at 214 $\mathrm{nm}, \theta_{214 \mathrm{~nm}}$, of S-1-Monostearoyglycerol in Hexane at $63.1^{\circ} \mathrm{C}$. 
in hexane at $63.1^{\circ} \mathrm{C}$. The value of $\theta_{214 \mathrm{~nm}}$ increases with increasing concentration but its increment gradually decreases. Linearity is not applicable between the $\theta_{214 \mathrm{~nm}}$ value and the concentration. Thus from the $\theta_{214 n m}$ value, we evaluated the difference in the molar absorptivity for left and right circularly polarized light, $\Delta \varepsilon$, using Equation 1.

$$
\Delta \varepsilon=33 \theta_{214 \mathrm{~nm}} / C d
$$

where $C$ denotes the concentration $\left(\mathrm{mol} \mathrm{dm}{ }^{-3}\right)$ and $d$, the light-path length.

CD spectra are generally influenced by the intensity change of the absorption band itself. Thus, for strict comparison of $\Delta \varepsilon$ at a band peak, normalization is necessary: we divided $\Delta \varepsilon$ by the molar absorption coefficient, $\varepsilon$, at the same wave length. The parameter $\Delta \varepsilon / \varepsilon$ is called as anisotropy factor, $g$.

Figure 7 shows the variation of $g$ against the concentration of $S$-1-monostearoylglycerol at $63.1^{\circ} \mathrm{C}$. The $g$ value decreases with an increase in the concentration of $S-1$ monostearoylglycerol. It is clear that the reduction in $g$ factor should be attributable to the decrease in $\Delta \varepsilon$ values. Why does the $g$ value decrease with an increase in the concentration?

Goto et al. reported the X-ray crystal structure of a single crystal of the $\beta_{1}$ ' form of $S$-1-monostearoylglycerol ${ }^{16)}$. They indicated that in the crystal of $S$-1-monostearoylglycerol the asymmetric unit contains two crystallographically independent molecules, which differ in the conformation of their glycerol parts. This is considered to be the influence of the hydrogen-bond network structures in the crystal. Consequently, $S$-1-monostearoylglycerol, although it exists as free monomers in its low concentrated solution, is thought to produce most probably two kinds of 1-monos-

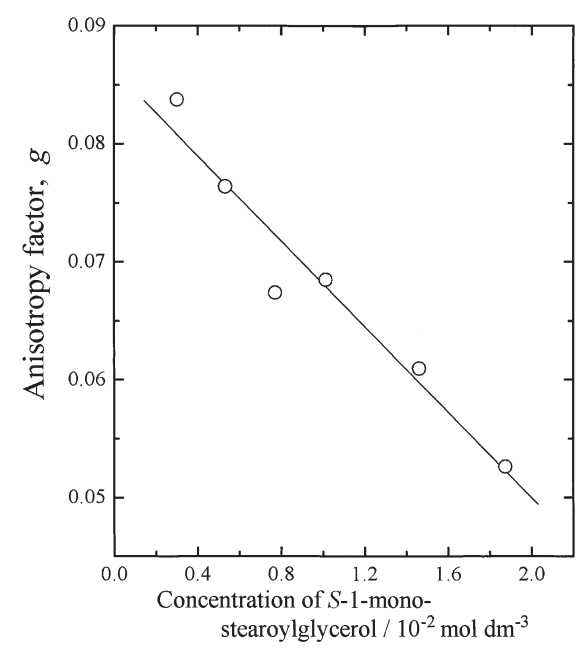

Fig. 7 Concentration Dependence of Anisotropy Factor, $g$, for $S$-1-Monostearoyglycerol in Hexane at $63.1^{\circ} \mathrm{C}$. tearoyglycerol having two distinct conformations in its high concentrated solution and combine with each other to form associated pairs, which have a less chirality (decreasing $\Delta \varepsilon$ ). Namely, the decrease in $g$ value (i.e., the decrease in chirality) for $S$-1-monostearoylglycerol with a rise of the concentration results from the association of chiral molecules by the hydrogen bonding; the association of chiral molecules is thought to reduce the overall chirality. More specifically, it is thought that a changing of magnetic dipole moment of $\left(n, \pi^{*}\right)$ transition of carbonyl group in glycerol, which affects CD intensity, is caused by the formation of hydrogen-bonding aggregate.

\section{CONCLUSION}

The hydrogen bonding of racemic 1-monostearoylglycerol in benzene was studied by comparison with that of chiral one through the ${ }^{1} \mathrm{H}$ NMR measurement. UV and circular dichroism (CD) measurements were carried out to know the effect of the concentration of $S$-1-monostearoyl glycerol on its chirality. The chemical shift of $\mathrm{OH}$ proton indicated that the hydrogen bonding between $R$ - and $S^{-}$ form molecules in $R S$-1-monoacylglycerol is stronger than that between $S$ - and $S$-form molecules in $S$-1-monoacylglycerol and that the difference in the strength of hydrogen-bonding between $R S$ - and $S$-form become to be small at high concentration. The chirality of $S$-1-monostearoylglycerol in hexane decreased with an increase in its concentration. This suggests that the two kinds of $S$-form molecules having different structures associate with each other in the concentrated solution. This association would decrease the overall chirality of $S$-1-monostearoylglycerol.

\section{References}

1. Chapman, D. The Polymorphism of Glycerides. Chem. Rev. 62, 433-456 (1962).

2. Maruyama, T.; Niiya, I.; Imamura, M.; Okada, M.; Matsumoto, T. Polymorphism of monoglycerides. II. Thermodynamic considerations on transition. Yukagaku 22, 85-88 (1973).

3. Maruyama, T.; Niiya, I.; Imamura, M.; Matsumoto, T. Study on polymorphism of monoglycerides. IV. Molecular chain length and thermodynamic values. Yukagaku 26, 104-109 (1977).

4. Lutton, E.S. Phases of saturated 1-monoglycerides C14-C22. J. Am. Oil Chem. Soc. 48, 78-781 (1971).

5. Larsson, K. Solid-state behavior of glycerides. Ark. Kemi 23, 35-56 (1964).

6. Larsson, K. Crystal structure of the L-1-monoglyceride of 11-bromoundecanoic acid. Acta Cryst. 21, 267-272 (1966). 


\section{H. Matsuzawa, K. Murakami and M. Iwahashi}

7. Eliel, E.L. Stereochemistry of Carbon compounds, McGraw-Hill Kogakusha. Tokyo. p. 44 (1962).

8. Tachibana, T.; Yoshizumi, T.; Hori, K. Monolayer Studies of Chiral and Racemic 12-Hydroxyoctadecanoic Acids. Bull. Chem. Soc. Jpn. 52, 34-41 (1979).

9. Polleck, J.R.A.; Stevens, R. Dictionary of Organic Compounds, Vol. 4. Eyre \& Spottishwoode Publishers. London. p. 2350 (1965).

10. Iwahashi, M.; Watanabe, Y.; Watanabe, T.; Muramatsu, M. Optically active and racemic glycerides. I. The thermodynamic and structural relationship between optically active and the corresponding racemic $\alpha$-monostearins in their crystalline, molten, and dissolved states. Bull. Chem. Soc. Jpn. 57, 1446-1448 (1984).

11. Iwahashi, M.; Ikumi, M.; Matsuzawa, H.; Moroi, Y.; Czarnecki, M.A.; Ozaki, Y. Molecular self-assembling of chiral and racemic butan-2-ol in carbon tetrachloride solutions. Vibrational Spectroscopy 20, 113-119 (1999).

12. Iwahashi, M.; Suzuki, M.; Katayama, N.; Matsuzawa, H.; Czarnecki, M. A.;Ozaki, Y.; Wakisaka, A. Molecular Self-Assembling of Butan-1-ol, Butan-2-ol, and 2-
Methylpropan-2-ol in Carbon Tetrachloride Solutions as Observed by Near-Infrared Spectroscopic Measurements. Applied Spectroscopy 54, 268-276 (2000).

13. Iwahashi, M.; Suzuki, M.; Czarnecki, M.A.; Liu, Y.; Ozaki, Y. Near-IR molar absorption coefficient for the OH-stretching mode of cis-9-octadecenoic acid and dissociation of the acid dimers in the pure liquid state. $J$. Chem. Soc., Faraday Trans. 91, 697-701 (1995).

14. Matsuzawa, H.; Minami, H.; Yano, H.; Wakabayashi, H.; Iwahashi, H.; Sakamoto, K.; Kaneko, D. Formation of Chiral Aggregates of Acylamino Acids in Organic Solvents. Studies in Surface Science and Catalysis 132, 137-140 (2001).

15. Iwahashi, M.; Matsuzawa, H.; Minami, H.; Yano, T.; T. Wakabayashi, T.; Ino, M.; Sasamoto, K. Solvent effect on chiral aggregate formation of acylamino acids. $J$. Oleo Sci. 51, 705-713 (2002).

16. Goto, M.; Kozawa, K.; Uchida, T. The crystal structure of the $\beta_{1}$ form of optically active $\alpha$-monostearin. Bull. Chem. So. Jpn. 61, 1434-1436 (1988). 